\title{
Minimally invasive treatment options for uterine fibroids - state-of-the art 2021
}

\author{
Jaroslaw Krzyzanowski ${ }^{1} \mathbb{D}$, Slawomir Wozniak ${ }^{1} \mathbb{D}$, Piotr Szkodziak ${ }^{1} \mathbb{C}^{\mathbb{D}}$, \\ Arkadiusz Krzyzanowski²], Wrona Wojciech ${ }^{1}\left[\right.$, Tomasz Paszkowski ${ }^{1}$ (]) \\ $13^{\text {rd }}$ Chair and Department of Gynecology, Medical University in Lublin, Poland \\ ${ }^{2}$ Chair and Department of Obstetrics and Pathology of Pregnancy, Medical University of Lublin, Poland
}

\begin{abstract}
Objectives: Uterine leyomyomas are benign, monoclonal tumors that can cause abnormal uterine bleeding, pelvic pain, dyspareunia and/or obstruction of bladder or rectum. Women's growing interest in treatments that avoid surgery and/or preserve the uterus has contributed to the development of minimally invasive methods.

Conducting a literature review and assessment of the effectiveness and safety of minimally invasive methods of treating fibroids, with particular emphasis on high intensity focused ultrasound.

Material and methods: Systematic review of MEDLINE, Cochrane and PubMed was performed using the following key words: uterine artery embolization, high-intensity focused ultrasound, microwave ablation, radiofrequency ablation, minimally invasive, leiomyoma, fertility, pregnancy. English abstracts relevant to the topic were selected. Full-text articles were carefully analyzed.

Results: Uterine artery embolization is a proven, widely accepted method that is effective in appropriately qualified cases. Although high focused ultrasound is still an experimental procedure, preliminary studies seem to be promising. If its efficacy and safety are confirmed in randomized controlled trials, this method may find its place in clinical practice. Microwave and radiofrequency ablation are interesting minimally invasive methods with the future potential to be recognized as a method of treating fibroids.

Conclusions: Minimally invasive methods are becoming an important treatment option for fibroids. Further research is needed to recognize these procedures as a fully-fledged alternative to surgical treatment.
\end{abstract}

Key words: fibroid; uterine artery embolization; high-intensity focused ultrasound; fertility

\section{INTRODUCTION}

Uterine leyomyomas are benign, monoclonal tumors originating from smooth muscle tissue. They consist of a large amount of extracellular substance, which contains fibronectin and proteoglycans. Myomas are surrounded by a pseudocapsule. It includes compressed muscle fibers, collagen fibers, neurofibers, and blood vessels [1].

The regulation of myoma growth is multifactorial. Its important elements are steroid hormones, growth factors, angiogenesis and apoptosis [2]. As they are estrogen and progesterone dependent tumors, they rarely occur before menarche and often decrease in size after menopause [3].

The risk factors for uterine fibroids include age, early menarche, low parity, obesity, diabetes, polycystic ovary syn- drome and African American race. Food and stimulants like alcohol or caffeine can also affect the prevalence of fibroids.

Due to the high percentage of undiagnosed fibroids, it is difficult to accurately determine their incidence. Depending on the population and diagnostic methods, studies show that 5.4 to $77 \%$ of women have fibroids [2].

About $30 \%$ of patients with myomas have symptoms like abnormal uterine bleeding, pelvic pain, dyspareunia and obstruction of bladder or rectum [3]. Myomas are also associated with about $10 \%$ of the cases of infertility and in 1-3\% of cases they are the only cause [4]. Asymptomatic myomas require only a medical surveillance, whereas symptomatic myomas are an indication for treatment.

\footnotetext{
Corresponding author:

Jaroslaw Krzyzanowski

$3^{\text {rd }}$ Chair and Department of Gynecology, Medical University in Lublin, 8 Jaczewskiego St, 20-954 Lublin, Poland

e-mail:jarek.krzyzanowski.94@gmail.com
} 
Currently used treatment methods include medical therapies [levonorgestrel intrauterine system, gonadotropin-releasing hormone $(\mathrm{GnRH})$ agonists], surgical treatment (myomectomy, hysterectomy) and minimally invasive procedures [5].

Women's growing interest in treatment options that avoid surgery and preserve the uterus has contributed to the development of minimally invasive methods such as: uterine artery embolization (UAE), magnetic resonance guided high-intensity focused ultrasound (MR-HIFU), ultrasound guided high-intensity focused ultrasound (US-HIFU), laparoscopic uterine artery occlusion (LUAO), microwave ablation and radiofrequency myolysis $[6,7]$.

\section{MATERIAL AND METHODS}

Systematic review of MEDLINE, Cochrane and PubMed was performed using the following keywords: uterine artery embolization, high-intensity focused ultrasound, microwave ablation, radiofrequency ablation, minimally invasive, leiomyoma, fertility, pregnancy. The review was performed in April 2021. English abstracts relevant to the topic were selected. Full-text articles written in English between 2008 and 2021 were carefully analyzed.

\section{Uterine artery embolization (UAE)}

UAE is a minimally invasive method that was initially used to reduce blood loss after myomectomy. In 1995, it was proposed as a separate treatment option for symptomatic fibroids in women wishing to preserve the uterus [8]. Over the years, a lot of research has been done on the UAE confirming its safety and effectiveness. The National Institute of Health and Clinical Excellence in its guidelines recognized UAE as an option of treatment for heavy menstrual bleeding [9]. However, despite more than 20 years of experience, this method still raises some controversy, especially when it comes to the qualification of women planning pregnancy in the future [10].

UAE is performed under local or epidural anesthesia by an interventional radiologist and takes about 30 to $60 \mathrm{~min}$ utes. This procedure is performed under fluoroscopy guidance. After percutaneous access is obtained (most often in the inguinal region), vascular catheter is inserted through the femoral artery into the aorta, internal iliac artery and uterine artery. After the correct position of the catheter is confirmed, an embolic agent is injected into the uterine artery along with a contrast agent. The injection continues until the flow in the uterine artery is blocked. The catheter is then removed, and a pressure dressing is applied to the puncture site. As UAE is associated with post-embolization pain, further treatment is necessary after the procedure. Patient controlled analgesia is the preferred choice [5].

UAE reduces the volume of fibroids by $25-60 \%$ and causes the resolution of heavy menstrual bleeding in $81-96 \%$, pelvic pain in $70-100 \%$ and pressure symptoms in $46-100 \%$ of patients.

UAE is considered a safe procedure. Possible complications include local side-effects associated with the angiography procedure, non-target embolization of other pelvic organs and infections [5].

Recent meta-analysis showed, that $40.5 \%$ of women wishing to conceive after UAE experienced at least one pregnancy. Finished pregnancies had an abortion rate of $28.6 \%$ while rate of preterm delivery, low birth weight and obstetrical complications were $12.8,10.0$ and $25.4 \%$ respectively. Although results of finished pregnancies are similar to general population, there was significant variability between included studies [11]. Furthermore, data exist showing that UAE is associated with an increased risk of complications such as miscarriage, placental adhesion, and uterine rupture [5]. UAE has been shown to have an adverse effect on the ovarian reserve, probably due to non-target embolization. Thus, patient concerns about their ovarian function should be considered contraindication to this procedure [12]. Before the procedure, patient should be informed that although, the incidence of leiomyosarcoma (LMS) is low and proper qualification (including endometrial biopsy and contrast enhanced magnetic resonance) has about $90 \%$ sensitivity in its detection, it is not possible to exclude a malignant lesion without histopathological examination. Therefore, embolization (and other minimally invasive treatments) may delay diagnosis and proper treatment [5]. Cost effectiveness of UAE seems to be on par with other treatment options [13].

Overall UAE is an effective procedure that improves the quality of life. However, precise qualification of the patient, taking into account the indications and contraindications, is necessary to obtain satisfactory results [5].

\section{High intensity focused ultrasound (HIFU)}

HIFU is a thermoablative treatment method for uterine fibroids. The myomas are heated transabdominally using focused ultrasound until complete ablation is confirmed. Constant magnetic resonance [14] or ultrasound [15] monitoring is required for this procedure. Before the procedure is performed, appropriate qualification is necessary: gynecological consultation with ultrasound examination and cytological smear, as well as radiological consultation, including MRI with contrast. The patient must be aware that, although proper qualification lowers the risk, it is not possible to exclude malignancy without histopathological examination [14].

The patient must show up for the treatment after overnight fasting. The abdomen must be shaved from the umbilicus to the superior border of the symphysis pubis. Skin must be free from lotions or creams, as even a small amount 
can cause skin burn during the procedure. Before the procedure, a vascular access is obtained and a catheter is inserted into the bladder [6]. There should be no bone or scar tissue in the sonication path, and the ultrasound focal region must cover most of the lesion [15]. In some cases filling the bladder and / or rectum may be necessary to shift the sonication beam from the sensitive area or to displace the loops of small intestine [6]. HIFU procedures are performed under intravenous sedation. Due to the need for effective communication with the patient during the procedure (as to prevent complications) deep sedation should be avoided [15].

According to several studies, HIFU is a relatively safe procedure. Complications occur in approximately $10-25 \%$ of cases with majority of them being minor (vaginal secretion, abdominal distention or lower abdominal pain that resolves spontaneously). Major complications (second or third degree skin burns, urinary retention, acute renal failure, intestinal perforation, hernia in abdominal wall) are rare [15, 16]. Hysterectomy and myomectomy have been shown to carry a much higher risk of serious complications [16]. In some cases, myoma calcification can alter the ultrasound beam and cause damage adjacent organs. Old surgical scars at the insonation site were found to increase the risk of severe skin burns [15].

Non-perfused volume (NPV) ratio is a key parameter correlating with the clinical outcomes of the procedure [17]. Achieving higher NPV ratio may contribute to the improvement of the effects of treatment. In 2004, when MR-HIFU was initially approved by the Food and Drug Administration (FDA), the guidelines allowed for a maximum of $50 \%$ NPV ratio. These guidelines changed in 2009, when ablation of $100 \%$ of the tumor volume became permissible. Park et al. [6] concluded, that achieving NPV ratio of $>80 \%$ results in a more significant reduction in tumor volume without increasing the complication rate. It even seems possible to achieve an NPV ratio of above $90 \%$ without affecting patient safety [18]. On the other hand, a low NPV ratio is associated with reduced therapeutic success and a higher rate of reintervention $[19,20]$. A study by lkink et al. found a seven-fold higher rate of reintervention after MR-HIFU compared to UAE with an NPV ratio of only $38 \%$ (26-62\%). The authors themselves noticed a much lower reintervention rate in the group of patients with NPV ratio of $>50 \%$ [19]. Increased thickness of the subcutaneous tissue of the anterior abdominal wall is one of the factors that make it difficult to achieve an appropriate NPV ratio [18].

Oxytocin, through its receptors in uterine smooth muscle cells, causes myometrial contraction and closure of blood vessels after delivery. A similar but weaker effect can be observed in non-pregnant uterus. Administration of oxytocin during the MR-HIFU procedure seems to reduce the sonication time and energy needed to achieve the set temperature. A greater reduction in tumor volume was also observed in the oxytocin group [21]. Similar results have been confirmed in other studies - both in the case of fibroids (MR-HIFU) [22] and adenomyosis (US-HIFU) [23].

Barnard et al. [20] in a randomized trial compared recovery trajectory after UAE and MR-HIFU. In the HIFU group, a lower percentage of the use of outpatient opioid medication and nonsteroidal anti-inflammatory drugs was found. Patients in this group returned to work faster than after UAE while in both groups this time was lower than after the hysterectomy. Two patients (10\%) in the HIFU group required another procedure within six weeks. In this study the mean NPV ratio was $<50 \%$, which may have caused the need of reintervention. However, exact data on these patients was not provided [20]. In a prospective study of 2,411 women treated with the US-HIFU, hysterectomy or myomectomy the Uterine Fibroid Symptom and Quality of Life (UFS-QOL) score improved faster after HIFU, however the absolute difference was small [16]. Among 272 women, the mean reduction in the volume of fibroids after US-HIFU was $58 \%, 66 \%$ and $77 \%$ at the 3, 6 and 12 months follow-up, respectively. At the same time, only in six cases the symptoms recurred. Furthermore US-HIFU was shown to be an effective treatment option for adenomyosis [24]. Another US-HIFU study involved 81 women with multiple (3-9) uterine fibroids. The UFS-QOL results improved significantly, although it should be noted that only 21 patients completed the six-month follow-up. During the follow-up two patients with submucosal myomas required myomectomy, as menstrual bleeding was not reduced [25]. There was also a significant decrease in myoma volume and an improvement in Symptom Severity Score (SSS) and UFS-QOL in this study [7].

Maintaining fertility is one of the reasons influencing the treatment decision, especially in case of minimally invasive procedures. For this reason, we believe that it is necessary to analyze the effect of the procedure on fertility and obstetric results. Unlike UAE [10], HIFU does not appear to affect ovarian reserve based on anti-Müllerian hormone (AMH) levels [7]. Similar results were obtained in women over the age of 40 [26]. Furthermore, pregnancy interval after HIFU was found to be significantly shorter than after myomectomy (10 and 13 months, respectively) [27]. The median time to pregnancy in the HIFU group was also shorter in another study (5.6 months) compared to the recommended recovery period of at least one year after myomectomy [28]. Several studies show, that it is possible to conceive and deliver healthy child after HIFU, but data from randomized studies in large study groups are lacking [27-30].

In a paper written by Juan Qin et al. [30] 24 out of 435 patients conceived spontaneously after US-HIFU. All seven patients who decided to continue their pregnancies were delivered on time, and the children received 8-10 points 
on the Apgar scale. In one case, an ongoing pregnancy was detected immediately after the procedure and abortion was performed due to the expected adverse effects of HIFU performed in the secretory phase of the menstrual cycle, when the patient was probably already pregnant [30]. Similar case of MR-HIFU performed on submucosal fibroid was presented by Polish team. In this case, the pregnancy was uneventful and the patient gave birth to a healthy child [29]. In our opinion care should be taken to ensure that procedures of this type take place in the preovulatory phase or are preceded by the determination of serum human chorionic gonadotropin (hCG).

Several studies presented outcomes of pregnancy after HIFU. The number of miscarriages and obstetric complications was comparable to that after myomectomy with majority of pregnancies ending at full term [27, 28, 31, 32]. In a study on 189 women who did not use contraception after US-HIFU, 131 conceived. Compared to the myomectomy, premature delivery rate was lower but other pregnancy complications as well as spontaneous abortions were on similar level. However, patients requiring re-intervention were excluded from this study which may have favorable for HIFU influence on the results [32]. Similar rates of pregnancies, live births, and obstetric complications in US-HIFU and myomectomy groups were confirmed by other retrospective study [27]. In another study 20 out of 276 (7.25\%) patients. became pregnant after MR-HIFU. No pregnancy related complications that could be associated with the procedure were detected [31]. Of the 406 treated patients, 78 (19.2\%) had a total of 80 pregnancies. 71 pregnancies ended with live birth, there were 3 miscarriages, 1 abortion and 5 on-going pregnancies. Three babies were born prematurely due to fetal distress, premature rupture of membranes and polyhydramnios [28].

Initial equipment purchase costs are high [33], however MR-HIFU can be a cost-effective alternative to established treatment options (UAE, myomectomy, hysterectomy) for symptomatic uterine fibroids. Its effectiveness depends on the number of procedures performed and the age of the patients. The NPV ratio has a high impact on the effectiveness of this procedure. As the NPV ratio increases, so does the cost per quality-adjusted life-year (QALY) gained and the potential savings. It is worth noting that this study did not take into account the patients' future reproductive plans [34]. In another study authors concluded, that myomectomy, UAE and MR-HIFU can be considered cost-effective over a five-year time frame [35].

\section{Microwave ablation}

ultrasound-guided percutaneous microwave ablation (PMWA) is a simple, minimally invasive procedure widely used in the treatment of tumors in organs other than the uterus. In this procedure, antennas with a microwave generator are placed in the tumor. In the case of larger tumors, it is possible to use several antennas to achieve higher ablation uniformity. lerardi et al. [36] concluded that PMWA is a safe and effective method of treating uterine fibroids. However, large randomized prospective trials are still needed to better demonstrate the benefits and drawbacks of this procedure [36].

Chinese researchers in a multicenter study on 311 patients achieved an ablation ratio of $86.6 \%$, a decrease in the volume of fibroids by $86.7 \%$ and a significant improvement in UFS-QOL. There were no serious complications. 27 (8.7\%) patients experienced abdominal pain that resolved within 12 hours and 19 (6.1\%) developed a small amount of vaginal discharge which resolved within 20 days. Overall, PMWA has been found to be a safe and effective treatment for uterine fibroids [37].

Tsuda and Kanaoka [38] presented the method of transcervical microwave ablation of submucosal fibroids. Out of 35 patients, only two required reintervention. In the follow-up, an increase in hemoglobin levels and a reduction in the volume of fibroids by $>70 \%$ was observed [38]. We believe that this method may be promising for patients with submucosal fibroids difficult to remove by hysteroscopy.

As it is with HIFU, oxytocin seems to decrease the heat sink during the microwave ablation, thus shortening the ablation time and increasing ablation rate [39]. Vasopressin administered between myometrium and myoma may play a similar role, but more research is needed to prove this [40].

We found no data regarding the cost-effectiveness or fertility outcomes of PMWA.

\section{Radiofrequency ablation (RFA)}

RFA is another ultrasound-guided procedure that allows the ablation of fibroids. The radiofrequency electrode is placed percutaneously or transvaginally with a needle guided into the selected myoma. The procedure is performed under ultrasound guidance and moderate intravenous anesthesia $[41,42]$. In a study on thirty five symptomatic patients that underwent percutaneous RFA, no major complications were reported and the severity of menstrual bleeding and pain was reduced. The most prevalent complications were: abdominal pain, erythema and skin reaction at entry site and symptoms of urinary tract infection [41]. In another study 19 patients underwent transvaginal RFA. There was significant improvement in QOL score after the procedure, while no major complications were observed. Overall, this method may be an alternative treatment option for symptomatic myomas, however prospective randomized trials are necessary to confirm its effectiveness and safety [42]. Data on the cost-effectiveness and pregnancy outcomes of this method were not found. 


\section{CONCLUSIONS}

Minimally invasive methods of treating fibroids are constantly evolving. UAE is a proven, widely accepted method that is effective in appropriately qualified cases. US and MR-guided HIFU, are still experimental methods. It seems that with a sufficiently high NPV ratio, HIFU may be safe and cost-effective.

Microwave and radiofrequency ablation are interesting minimally invasive techniques that have the potential to be recognized as a method of treating fibroids in the future, provided that the initial results are confirmed by randomized control trials.

\section{Funding}

Funds allocated to statutory activities of the 3rd Chair and Department of Gynecology, Medical University of Lublin.

\section{Conflict of interest}

None.

\section{REFERENCES}

1. Tinelli A, Sparic R, Kadija S, et al. Myomas: anatomy and related issues. Minerva Ginecol. 2016; 68(3): 261-273, indexed in Pubmed: 26785282.

2. Sparic R, Mirkovic L, Malvasi A, et al. Epidemiology of uterine myomas: a review. Int J Fertil Steril. 2016; 9(4): 424-435, indexed in Pubmed: 26985330.

3. Williams ARW. Uterine fibroids - what's new? F1000Res. 2017; 6: 2109, doi: 10.12688/f1000research.12172.1, indexed in Pubmed: 29259779.

4. Al-Hendy A, Myers ER, Stewart E. Uterine fiibroids: burden and unmet medical need. Semin Reprod Med. 2017; 35(6): 473-480, doi: 10.1055/s 0037-1607264, indexed in Pubmed: 29100234.

5. Szkodziak P, Pyra K, Szkodziak F, et al. The Lublin Protocol of the uterine arteries embolization in the treatment of symptomatic uterine fibroids. J Vis Exp. 2020(163), doi: 10.3791/61530, indexed in Pubmed: 33016950.

6. Park MJ, Kim Ys, Rhim H, et al. Safety and therapeutic efficacy of complete or near-complete ablation of symptomatic uterine fibroid tumors by MR imaging-guided high-intensity focused US therapy. J Vasc Interv Radiol. 2014; 25(2): 231-239, doi: 10.1016/j.jvir.2013.11.011, indexed in Pubmed: 24360886

7. Lee JS, Hong GY, Lee KH, et al. Changes in anti-müllerian hormone levels as a biomarker for ovarian reserve after ultrasound-guided high-intensity focused ultrasound treatment of adenomyosis and uterine fibroid. BJOG. 2017; 124(Suppl 3): 18-22, doi: 10.1111/1471-0528.14739, indexed in Pubmed: 28856867.

8. Ravina JH, Ciraru-Vigneron N, Bouret JM, et al. Arterial embolisation to treat uterine myomata. Lancet. 1995; 346(8976): 671-672, doi: 10.1016/s0140-6736(95)92282-2.

9. Heavy menstrual bleeding: assessment and management. https://www nice.org.uk/guidance/ng88 (May 24, 2021).

10. Chen HT, Athreya S. Systematic review of uterine artery embolisation practice guidelines: are all the guidelines on the same page? Clin Radiol. 2018; 73(5): 507.e9-507.e15, doi: 10.1016/j.crad.2017.12.005, indexed in Pubmed: 29352594

11. Ghanaati $H$, Sanaati M, Shakiba M, et al. Pregnancy and its outcomes in patients after uterine fibroid embolization: a systematic review and meta-analysis. Cardiovasc Intervent Radiol. 2020;43(8): 1122-1133, doi: 10.1007/s00270-020-02521-6, indexed in Pubmed: 32458009.

12. Czuczwar $P$, Stepniak $A$, Milart $P$, et al. Comparison of the influence of three fibroid treatment options: supracervical hysterectomy, ulipristal acetate and uterine artery embolization on ovarian reserve - an observational study. J Ovarian Res. 2018; 11(1): 45, doi: 10.1186/s13048-0180420-1, indexed in Pubmed: 29859107.
13. Wang C, Kuban JD, Lee SR, et al. Utilization of endovascular and surgical treatments for symptomatic uterine leiomyomas: a population health perspective. J Vasc Interv Radiol. 2020; 31(10): 1552-1559.e1, doi: 10.1016/j.jvir.2020.04.039, indexed in Pubmed: 32917502.

14. Kröncke T, David M. MR-guided focused utrasound in fibroid treatment - results of the 4th Radiological-Gynecological Expert Meeting. Rofo. 2019; 191(7): 626-629, doi: 10.1055/a-0884-3143, indexed in Pubmed: 31137044.

15. Chen J, Chen W, Zhang L, et al. Safety of ultrasound-guided ultrasound ablation for uterine fibroids and adenomyosis: a review of 9988 cases. Ultrason Sonochem. 2015; 27: 671-676, doi: 10.1016/j. ultsonch.2015.05.031, indexed in Pubmed: 26093678.

16. Chen J, Li Y, Wang Z, et al. Committee of the Clinical Trial of HIFU versus Surgical Treatment for Fibroids. Evaluation of high-intensity focused ultrasound ablation for uterine fibroids: an IDEAL prospective exploration study. BJOG. 2018; 125(3): 354-364, doi: 10.1111/1471-0528.14689, indexed in Pubmed: 28421665.

17. Lozinski T, Filipowska J, Pyka M, et al. Magnetic resonance-guided high-intensity ultrasound (MR-HIFU) in the treatment of symptomatic uterine fibroids - five-year experience. Ginekol Pol. 2021 [Epub ahead of print], doi: 10.5603/GP.a2021.0098, indexed in Pubmed: 33914323.

18. Keserci B, Duc NM. Magnetic resonance imaging parameters in predicting the treatment outcome of high-intensity focused ultrasound ablation of uterine fibroids with an immediate nonperfused volume ratio of at least 90. Acad Radiol. 2018; 25(10): 1257-1269, doi: 10.1016/j. acra.2018.01.022, indexed in Pubmed: 29525424.

19. Ikink ME, Nijenhuis RJ, Verkooijen HM, et al. Volumetric MR-guided high-intensity focused ultrasound versus uterine artery embolisation for treatment of symptomatic uterine fibroids: comparison of symptom improvement and reintervention rates. Eur Radiol. 2014; 24(10): 2649-2657, doi: 10.1007/s00330-014-3295-6, indexed in Pubmed: 25117743.

20. Barnard EP, AbdElmagied AM, Vaughan LE, et al. Periprocedural outcomes comparing fibroid embolization and focused ultrasound: a randomized controlled trial and comprehensive cohort analysis. Am J Obstet Gynecol. 2017; 216(5): 500.e1-500.e11, doi: 10.1016/j.ajog.2016.12.177, indexed in Pubmed: 28063909.

21. Bakry MA, Fawzi MM, Shaban MH. Oxytocin administration improves efficiency of HIFU treatment of uterine fibroids. J Anesth Clin Res. 2020; 11(3): 1000943, doi: 10.35248/2155-6148.20.11.943.

22. Lozinski T, Filipowska J, Krol P, et al. Oxytocin administration in high-intensity focused ultrasound treatment of myomata. Biomed Res Int. 2018; 2018: 7518026, doi: 10.1155/2018/7518026, indexed in Pubmed: 30057909.

23. Wu Q, He M, Wang J, et al. Effects of oxytocin on high intensity focused ultrasound (HIFU) ablation of adenomysis: a prospective study. Eur J Radiol. 2014, doi: 10.1016/j.ejrad.2014.05.008.

24. Lee JS, Hong GY, Park BJ, et al. Ultrasound-guided high-intensity focused ultrasound treatment for uterine fibroid \& adenomyosis: A single center experience from the Republic of Korea. Ultrason Sonochem. 2015; 27:682687, doi: 10.1016/j.ultsonch.2015.05.033, indexed in Pubmed: 26072367.

25. He M, Jacobson $\mathrm{H}$, Zhang C, et al. A retrospective study of ultrasound-guided high intensity focussed ultrasound ablation for multiple uterine fibroids in South Africa. Int J Hyperthermia. 2018; 34(8): 1304-1310, doi: 10.1080/02656736.2017.1421323, indexed in Pubmed: 29278943.

26. Cheung VYT, Lam TPW, Jenkins $C R$, et al. Ovarian reserve after ultrasound-guided high-intensity focused utrasound for uterine fibroids: preliminary experience. J Obstet Gynaecol Can. 2016; 38(4): 357-361, doi: 10.1016/j.jogc.2016.02.006, indexed in Pubmed: 27208605.

27. Jiang Z, Li Q, Li W, et al. A comparative analysis of pregnancy outcomes of patients with uterine fibroids after high intensity focused ultrasound ablation and laparoscopic myomectomy: a retrospective study. Int J Hyperthermia. 2021; 38(1): 79-84, doi: 10.1080/02656736.2021.1874547, indexed in Pubmed: 33478288

28. Zou M, Chen L, Wu C, et al. Pregnancy outcomes in patients with uterine fibroids treated with ultrasound-guided high-intensity focused ultrasound. BJOG. 2017; 124(Suppl 3): 30-35, doi: 10.1111/1471-0528.14742, indexed in Pubmed: 28856864

29. Łoziński T, Filipowska J, Ludwin A, et al. The outcome of magnetic resonance-guided high-intensity ultrasound for clinically symptomatic submucosal uterine fibroid performed accidentally in very early pregnancy: a case report. Int J Hyperthermia. 2019; 36(1): 975-979, doi: 10.1080/02656736.2019.1660002, indexed in Pubmed: 31542975.

30. Qin J, Chen JY, Zhao WP, et al. Outcome of unintended pregnancy after ultrasound-guided high-intensity focused ultrasound ablation of uterine 
fibroids. Int J Gynaecol Obstet. 2012; 117(3): 273-277, doi: 10.1016/j. ijgo.2012.01.011, indexed in Pubmed: 22465558.

31. Łoziński T, Filipowska J, Gurynowicz G, et al. The effect of high-intensity focused ultrasound guided by magnetic resonance therapy on obstetrical outcomes in patients with uterine fibroids - experiences from the main Polish center and a review of current data. Int J Hyperthermia. 2019; 36(1): 582-590, doi: 10.1080/02656736.2019.1616117, indexed in Pubmed: 31159642

32. Li JS, Wang Y, Chen JY, et al. Pregnancy outcomes in nulliparous women after ultrasound ablation of uterine fibroids: A single-central retrospective study. Sci Rep. 2017; 7(1): 3977, doi: 10.1038/s41598-017-04319-y, indexed in Pubmed: 28638108.

33. Łoziński T, Filipowska J, Gurynowicz G, et al. Non-invasive therapeutic use of high-intensity focused ultrasound (HIFU) with 3 tesla magnetic resonance imaging in women with symptomatic uterine fibroids. Ginekol Pol. 2017; 88(9): 497-503, doi: 10.5603/GP.a2017.0091, indexed in Pubmed: 29057436.

34. Zowall H, Cairns JA, Brewer $\mathrm{C}$, et al. Cost-effectiveness of magnetic resonance-guided focused ultrasound surgery for treatment of uterine fibroids. BJOG. 2008; 115(5): 653-662, doi: 10.1111/j.1471-0528.2007.0 1657.x, indexed in Pubmed: 18333948.

35. Moriarty P, Elizabeth A. Cost-effectiveness of uterine-preserving procedures for the treatment of uterine fibroid symptoms in the USA.J Comp Eff Res. 2014; 3: 503-514.

36. lerardi AM, Savasi V, Angileri SA, et al. Percutaneous high frequency microwave ablation of uterine fibroids: systematic review. Biomed
Res Int. 2018; 2018: 2360107, doi: 10.1155/2018/2360107, indexed in Pubmed: 29511672

37. Liu H, Zhang J, Han ZY, et al. Effectiveness of ultrasound-guided percutaneous microwave ablation for symptomatic uterine fibroids: a multicentre study in China. Int J Hyperthermia. 2016; 32(8): 876-880, doi: 10.1080/02656736.2016.1212276, indexed in Pubmed: 27405972.

38. Tsuda A, Kanaoka Y. Outpatient transcervical microwave myolysis assisted by transabdominal ultrasonic guidance for menorrhagia caused by submucosal myomas. Int J Hyperthermia. 2015; 31(6): 588-592, doi: 10.3109/02656736.2015.1036385, indexed in Pubmed: 26287991.

39. Fu Y, Feng Q, Zhang S, et al. Application of oxytocin in ultrasound-guided percutaneous microwave ablation for treatment of hypervascular uterine fibroids: a preliminary report. Int J Hyperthermia. 2019; 36(1): 761-767, doi: 10.1080/02656736.2019.1639832, indexed in Pubmed: 31431080.

40. Tsuda A, Kanaoka Y. Submyometrial vasopressin injection before microwave ablation of vascular-rich submucosal myomas: a preliminary case study. Int J Hyperthermia. 2019; 36(1): 739-743, doi: 10.1080/02656736.2019.1612102, indexed in Pubmed: 31385549.

41. Yüce G, Tayarer A, Keskin HL, et al. Ultrasound guided percutaneous radiofrequency thermal ablation of symptomatic uterine fibroids - results from a single center and 52 weeks of follow up. Ginekol Pol. 2020; 91(8): 447-452, doi: 10.5603/GP.2020.0074, indexed in Pubmed: 32902841.

42. Turtulici G, Orlandi D, Dedone G, et al. Ultrasound-guided transvaginal radiofrequency ablation of uterine fibroids assisted by virtual needle tracking system: a preliminary study. Int J Hyperthermia. 2019; 35(1): 97-104, doi: 10.1080/02656736.2018.1479778, indexed in Pubmed: 30012030. 